\title{
Expression and clinical significance of HMGB1 in human liver cancer: Knockdown inhibits tumor growth and metastasis in vitro and in vivo
}

\author{
YA-DONG DONG, LONG CUI, CHENG-HONG PENG, DONG-FENG CHENG, BAO-SAN HAN and FANG HUANG
}

Department of General Surgery, Rui Jin Hospital, Shanghai Jiao Tong University

School of Medicine, Shanghai 200025, P.R. China

Received April 17, 2012; Accepted August 21, 2012

DOI: $10.3892 / o r .2012 .2070$

\begin{abstract}
The high-mobility group box 1 (HMGB1) signaling pathway plays a crucial role in tumorigenesis and progression of many malignant cancers. The present study aimed to investigate the expression and clinical significance of HMGB1 in human primary liver cancer, and further explore the molecular mechanisms of HMGB1 in tumor growth and metastasis. Forty cases of human liver cancer and normal liver tissues were collected. The expression of HMGB1 was assessed using RT-PCR and western blot assays in biopsy samples. The HMGB1 pathway in vitro was blocked using transfection of the recombinant small hairpin RNA adenovirus vector rAd5HMGB1 into the human liver cancer cell line SMMC-7721. The expression of HMGB1, phosphorylated AKT (p-AKT), Ki-67 and matrix metallopeptidase-2 (MMP-2) was detected by Real-PCR and western blot assays. Cell proliferative activities and metastatic capability were determined by MTT and Transwell assays. Cell cycle distribution and apoptosis were detected by flow cytometry. A subcutaneous xenograft tumor model was established, validating the effects of rAd5-HMGB1 on tumor growth in vivo. As a consequence, HMGB1 was found to be highly expressed in liver cancer compared with normal tissues, and was positively associated with pathological grade and distant metastases of liver cancer. Knockdown of HMGB1 downregulated the expression of p-AKT, Ki-67 and MMP-2, inhibited the proliferative activities and metastatic potential of SMMC-7721 cells, induced cell cycle arrest and apoptosis, and slowed the growth of xenograft tumors. Altogether, the expression of HMGB1 is closely correlated with pathological grade and distant metastases of liver cancer, and knockdown of
\end{abstract}

Correspondence to: Professor Cheng-Hong Peng and Dr Dong-Feng Cheng, Department of General Surgery, Rui Jin Hospital, Shanghai Jiao Tong University School of Medicine, Rui Jin 2nd Road, Luwan, Shanghai 200025, P.R. China

E-mail: pengchh@sina.cn

E-mail: chengdf1059@163.com

Key words: high-mobility group box 1, protein kinase B, liver cancer, RNA interference, growth, metastasis
HMGB1 inhibits liver cancer growth and metastasis, suggesting that HMGB1 may be involved in liver cancer development and progression through AKT-mediated regulation of Ki-67 and MMP-2 expression, and represent a potential therapeutic target for this aggressive malignancy.

\section{Introduction}

Liver cancer in men is the fifth most frequently diagnosed cancer worldwide but the second most frequent cause of cancer death. In women, it is the seventh most commonly diagnosed cancer and the sixth leading cause of cancer death. An estimated 748,300 new liver cancer cases and 695,900 cancer deaths occurred worldwide in 2008 (1). Half of these cases and deaths are estimated to occur in China (2). Liver cancer is also a genetic disease developing from a multi-step process. Single or multiple mutations in genes related to growth control, invasion and metastasis form the molecular genetic basis of malignant transformation and tumor progression (3). Therefore, identification of key genes and targets in signaling pathways related to tumorigenesis is indispensible for the diagnosis and prevention of liver cancer.

High mobility group box 1 (HMGB1) is a DNA-binding nuclear protein, released actively following cytokine stimulation as well as passively during cell death. It is the prototypic damage-associated molecular pattern (DAMP) molecule and has been implicated in several inflammatory disorders (4). HMGB1 interacts with its receptors RAGE and TLR that belong to family of pattern recognition receptors and involves in activation of pathways leading to production of proinflammatory cytokines, forming a positive feedback circuit of inflammation. Serum HMGB-1 increases after major gastrointestinal surgery, and its peak levels correlate with the duration of SIRS and postoperative pulmonary dysfunction. It has been revealed in mediation of sepsis and represents a potential target in therapy of various disorders related to inflammation (5-7). Moreover, HMGB1 is a nuclear protein that binds to a number of molecules related to cancer and associates with each of the hallmarks of cancer including unlimited replicative potential, ability to develop angiogenesis, evasion of apoptosis, self-sufficiency in growth signals, insensitivity to inhibitors of growth, inflammation, tissue invasion and metastasis $(8,9)$. 
It has been reported that HMGB1 and its receptor RAGE are expressed in many malignant tumors (10). Increased expression of HMGB1 is associated with progression and poor prognosis in human nasopharyngeal carcinoma (11). It is also a useful serological biomarker for early diagnosis as well as evaluating the tumorigenesis, stage, and prognosis of gastric cancer (12). HMGB1 protein may contribute to the malignant progression of head and neck cell carcinoma, and present as a novel prognostic marker and a potential therapeutic target for cancer (13).

HMGB1 pathway plays an important role in the metastasis of multiple cancers. They are closely associated with metastasis of squamous cell carcinoma (14). HMGB1 secreted from primary tumors spread to the regional lymph nodes decreases the number of macrophages to attenuate the anti-metastatic defense of the lymph nodes in patients with colorectal cancer $(15,16)$. It can activate TLR4 and RAGE signaling pathways to induce caspase-1 with subsequent production of many inflammatory mediators which in turn promotes cancer invasion and metastasis (17). Hypoxia-increased RAGE expression also regulates tumor cell invasion and metastasis through activation of Erk1/2, AKT and nuclear translocation of NF- $\kappa \mathrm{B}$ (18). Targeting HMGB1 inhibits ovarian cancer growth and metastasis by RNA interference (RNAI), Therefore, HMGB1 is a newly identified gene associated with cancer growth and metastasis, representing a new therapeutic target for the treatment of cancer (19).

Recently, it has been shown that reduced HMGB1 expression induced by RNAI inhibits the bioactivity of hepatic carcinoma cells (20). However, the expression and clinical significance of HMGB1 in liver cancer, especially the molecular mechanisms of HMGB1 in tumorigenesis of liver cancer have rarely been reported. In the present study, human liver cancer and normal liver tissues were collected. The expression of HMGB1 was assessed using RT-PCR and western blot assays in biopsy samples. Using a loss of function approach, we investigated in vitro and in vivo the role of HMGB1 signaling pathway in growth and metastasis of liver cancer, and attempted to find a promising therapeutic target for liver cancer.

\section{Materials and methods}

Materials. SMMC-7721 cell line used in the experiment was from Institute of Biochemistry and Cell Biology (Shanghai, China); six-week-old female immune-deficient nude mice (BALB/c-nu) were purchased from Shanghai SLAC Laboratory Animal Co., Ltd. (Shanghai Laboratory Animal Center of Chinese Academy Sciences). Adenovirus-mediated HMGB1 small hairpin RNA vector, negative control vector and virionpackaging elements were from Genechem (Shanghai, China). The primers of HMGB1, p-AKT, Ki-67 and MMP-2 were synthesized by ABI Co., Ltd. (USA). All antibodies were from Santa Cruz Biotechnology (Santa Cruz, CA, USA).

Drugs and reagents. 3-(4,5)-Dimethylthiahiazo(-z-yl)-3,5-diphenytetrazolium bromide (MTT) was from Dingguo biology (Shanghai, China); Dulbecco's modified Eagle's medium (DMEM) and fetal bovine serum (FBS) were from Thermo Fisher Scientific Inc. (Waltham, MA, USA); TRIzol Reagent and Lipofectamine 2000 were from Invitrogen (Carlsbad, CA, USA); M-MLV Reverse Transcriptase was from Promega (Madison, WI, USA); SYBR Green Master Mix was from
Takara (Otsu, Japan); Cell cycle analysis kit and apoptosis kit (Propidium Iodide (PI), RNase A, Annexin V-FITC) were from KeyGen Biology (Nanjing, China). ECL-PLUS/kit was from GE Healthcare (Piscataway, NJ, USA).

Tissue samples. Forty freshly resected liver cancer and normal liver tissue samples were collected at the Department of General Surgery of Shanghai Rui Jin Hospital during 2010 and were classified according to American Joint Committee on Cancer (AJCC) TNM staging system. Tissues and clinical information were obtained as part of an approved study at Shanghai Jiao Tong University School of Medicine. There were 30 cases of liver cancer tissues and 10 cases of normal liver tissues. A portion of each tissue sample was stored in liquid nitrogen for RT-PCR and western blot examination. All tumors and normal tissues were diagnosed by two independent gastroenterologists.

$R T-P C R$. To quantitatively determine the mRNA expression level of HMGB1, p-AKT, Ki-67 and MMP-2 in liver cancer, normal liver tissues and SMMC-7721 cells, RT-PCR was used. Total RNA of each clone was extracted with TRIzol according to the manufacturer's protocol. Reverse-transcription was carried out using M-MLV and cDNA amplification was carried out using SYBR Green Master Mix kit according to the manufacturer's protocol. The genes were amplified using specific oligonucleotide primer and human glyceraldehyde-3-phosphate dehydrogenase (GAPDH) gene was used as an endogenous control. The PCR primer sequences were: HMGB1, 5'-ATA TGGCAAAAGCGGACAAG-3' and 5'-AGGCCAGGATGTT CTCCTTT-3'; p-AKT, 5'-GGAGAUCAUGCAGCAUCGC dtdt-3' and 5'-GCGAUGCUGCAUGAUCUCCdtdt-3'; Ki-67, 5'-CTTTGGGTGCGACTTGACG-3' and 5'-GTCGACCC CGCTCCTTTT-3'; MMP-2, 5'-GGCCCTGTCACTCCTGA GAT-3' and 5'-GGCATCCAGGTTATCGGGGA-3'; GAPDH, 5'-CAACGAATTTGGCTACAGCA-3' and 5'-AGGGGTCTA CATGGCAACTG-3'. Data were analyzed using the comparative $\mathrm{Ct}$ method $\left(2^{-\Delta \Delta \mathrm{Ct}}\right)$. Three separate experiments were performed for each clone.

Western blot assay. Liver cancer, normal liver tissues and SMMC-7721 cells were harvested and extracted using lysis buffer (Tris-HCl, SDS, mercaptoethanol, glycerol). Cell extracts were boiled for $5 \mathrm{~min}$ in loading buffer and then equal amount of cell extracts were separated on $15 \%$ SDS-PAGE gels. Separated protein bands were transferred into polyvinylidene fluoride (PVDF) membranes and the membranes were blocked in $5 \%$ skim milk powder. The primary antibodies against HMGB1, p-AKT, Ki-67 and MMP-2 were diluted according to the instructions of antibodies and incubated overnight at $4^{\circ} \mathrm{C}$. Then, horseradish peroxidase-linked secondary antibodies were added at a dilution ratio of 1:1000, and incubated at room temperature for $2 \mathrm{~h}$. The membranes were washed with PBS for three times and the immunoreactive bands were visualized using ECL-PLUS/Kit according to the manufacturer's instructions. The relative protein level in different cell lines was normalized to GAPDH concentration. Three separate experiments were performed for each clone.

Cell culture and adenovirus transfection. SMMC-7721 cells were cultured in DMEM medium supplemented with $10 \%$ 
heat-inactivated FBS, $100 \mu \mathrm{g} / \mathrm{ml}$ of penicillin and $100 \mu \mathrm{g} / \mathrm{ml}$ of streptomycin. They were all placed in a humidified atmosphere containing $5 \% \mathrm{CO}_{2}$ at $37^{\circ} \mathrm{C}$. Recombinant adenovirus vector rAd5-HMGB1 and negative control rAd5-GFP were transfected into SMMC-7721 cells. Cells were subcultured at a 1:5 dilution in $300 \mu \mathrm{g} / \mathrm{ml} \mathrm{G} 418$-containing medium. Positive stable transfectants were selected and expanded for further study. The clone in which the rAd5-HMGB1 virus vectors transfected was named as rAd5-HMGB1 group, the negative control vectors transfected was named as GFP group and SMMC-7721 cells were named as CON group.

Cell proliferation assay. Cell proliferation was analyzed with the MTT assay. Briefly, cells infected with rAd5-HMGB1 were incubated in 96-well-plates at a density of $1 \times 10^{5}$ cells per well with DEME medium supplemented with $10 \%$ FBS. Cells were treated with $20 \mu \mathrm{l}$ MTT dye at $0,24,48,72 \mathrm{~h}$ and then incubated with $150 \mu \mathrm{l}$ of DMSO for $5 \mathrm{~min}$. The color reaction was measured at $570 \mathrm{~nm}$ with enzyme immunoassay analyzer (Bio-Rad, Hercules, CA, USA). The proliferation activity was calculated for each clone.

Transwell invasion assay. Transwell filters were coated with matrigel $(3.9 \mu \mathrm{g} / \mu \mathrm{l}, 60-80 \mu \mathrm{l})$ on the upper surface of a polycarbonic membrane (diameter $6.5 \mathrm{~mm}$, pore size $8 \mu \mathrm{m}$ ). After incubating at $37^{\circ} \mathrm{C}$ for $30 \mathrm{~min}$, the matrigel solidified and served as the extracellular matrix for analysis of tumor cell invasion. Harvested cells $\left(1 \times 10^{5}\right)$ in $100 \mu$ of serum-free DMEM were added into the upper compartment of the chamber. A total of $200 \mu \mathrm{l}$ conditioned medium derived from NIH3T3 cells was used as a source of chemoattractant, and was placed in the bottom compartment of the chamber. After $24 \mathrm{~h}$ of incubation at $37^{\circ} \mathrm{C}$ with $5 \% \mathrm{CO}_{2}$, the medium was removed from the upper chamber. The non-invaded cells on the upper side of the chamber were scraped off with a cotton swab. The cells that had migrated from the matrigel into the pores of the inserted filter were fixed with $100 \%$ methanol, stained with hematoxylin, and mounted and dried at $80^{\circ} \mathrm{C}$ for $30 \mathrm{~min}$. The number of cells invading through the matrigel was counted in three randomly selected visual fields from the central and peripheral portion of the filter using an inverted microscope (magnification, x200). Each assay was repeated three times.

Cell apoptosis analysis. To detect cell apoptosis, cells infected with rAd5-HMGB1 were trypsinized, washed with cold PBS and resuspended in binding buffer according to the instruction of the apoptosis kit. FITC-Annexin V and PI were added to the fixed cells for $20 \mathrm{~min}$ in darkness at room temperature. Then, Annexin $\mathrm{V}$ binding buffer was added to the mixture before the fluorescence was measured on FACSort flow cytometer. The cell apoptosis was analyzed using the CellQuest software (Becton Dickinson, USA). Three separate experiments were performed for each clone.

Cell cycle analysis. To detect cell cycle variation, cells infected with rAd5-HMGB1 were trypsinized, washed by PBS and fixed with $80 \%$ cold ethanol overnight at $-20^{\circ} \mathrm{C}$. After PBS washing, the fixed cells were stained with PI in the presence of RNase A for $30 \mathrm{~min}$ at room temperature in darkness. Each sample was filtered through a $50 \mu \mathrm{m}$ nylon filter to obtain

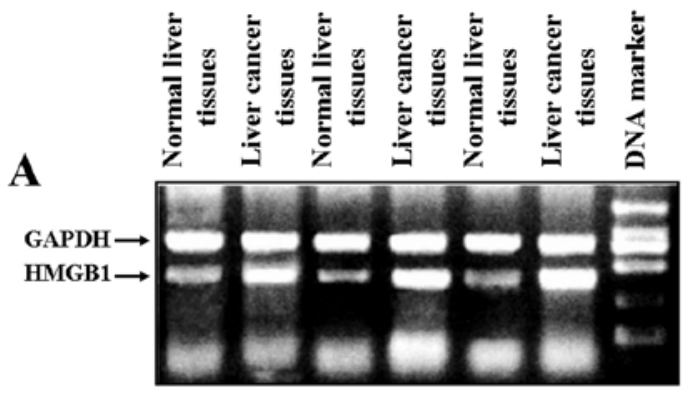

B

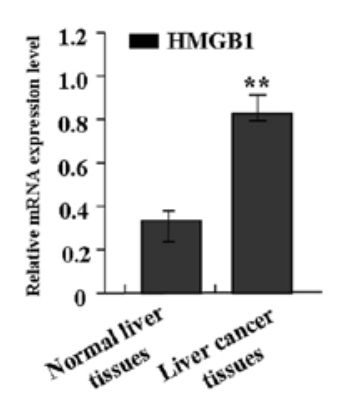

C

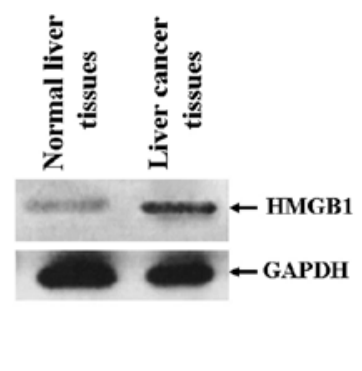

Figure 1. The expression of HMGB1 in human liver cancer evaluated by RT-PCR and western blot assays. (A and B) The mRNA expression level of HMGB1 was significantly increased in liver cancer tissues in comparison with normal liver tissues $\left({ }^{* *} \mathrm{P}<0.01\right)$. (C) The protein expression level of HMGB1 was significantly increased in liver cancer tissues compared with the normal liver tissues.

single-cell suspension. The samples were then analyzed on FACsort flow cytometer (Becton Dickinson, Mountain View, CA, USA). ModFit3.0 software (Verity Software House, Topsham, ME, USA) was used for cell cycle analysis. Three separate experiments were performed for each clone.

In vivo tumor xenograft studies. Two mice were injected subcutaneously with $1 \times 10^{8}$ SMMC-7721 cells in $50 \mu 1$ of PBS pre-mixed with an equal volume of matrigel matrix (Becton Dickinson). Mice were monitored daily, and two mice developed subcutaneous tumors. When the tumor size reached $\sim 5 \mathrm{~mm}$ in length, they were surgically removed, cut into $1-2 \mathrm{~mm}^{3}$ pieces, and re-seeded individually into 12 other mice. When tumor size reached $\sim 5 \mathrm{~mm}$ in length, the mice were randomly assigned to control (CON), GFP and rAd5-HMGB1 groups. In GFP and rAd5-HMGB1 groups, $15 \mu \mathrm{l}$ of adenovirus was injected into subcutaneous tumors using a multi-site injection format. Mice in CON group received $15 \mu \mathrm{l}$ of PBS only. Injections were repeated on the third day after initial treatment. The tumor volume every three days was measured with a caliper, using the formula volume $=(\text { length } \mathrm{x} \text { width })^{2} / 2$.

Statistical analysis. The result of each experiment was shown as mean \pm SD when applicable. Statistically significant difference in each assay was determined by SPSS version 11.5. Difference in each group was tested for significance using $\mathrm{t}$-test and ANOVA analysis of variance. $\mathrm{P}<0.05$ was considered significant.

\section{Results}

Expression of HMGB1 in human liver cancer. The expression of HMGB1 in liver cancer was evaluated using RT-PCR and 
Table I. The correlation of HMGB1 expression with clinicopathologic characteristics of human liver cancer.

\begin{tabular}{|c|c|c|c|}
\hline $\begin{array}{l}\text { Clinicopathological } \\
\text { factors }\end{array}$ & $\mathrm{n}$ & $\begin{array}{l}\text { HMGB1 mRNA } \\
\text { expression } \\
(\text { mean } \pm \text { SD })\end{array}$ & $\mathrm{P}$ \\
\hline \multicolumn{4}{|l|}{ Age } \\
\hline$>60$ & 11 & $0.82 \pm 0.35$ & \\
\hline$\leq 60$ & 19 & $0.84 \pm 0.19$ & $>0.05$ \\
\hline \multicolumn{4}{|l|}{ Pathological grade } \\
\hline $\mathrm{I}+\mathrm{II}$ & 9 & $0.49 \pm 0.16$ & \\
\hline III+IV & 21 & $0.92 \pm 0.55$ & $<0.01$ \\
\hline \multicolumn{4}{|l|}{ Pathological type } \\
\hline Massive type & 22 & $0.81 \pm 0.24$ & \\
\hline Nodular type & 8 & $0.84 \pm 0.32$ & $>0.05$ \\
\hline \multicolumn{4}{|l|}{ AFP } \\
\hline$\leq 400 \mu \mathrm{g} / \mathrm{l}$ & 9 & $0.80 \pm 0.17$ & \\
\hline$>400 \mu \mathrm{g} / 1$ & 21 & $0.83 \pm 0.26$ & $>0.05$ \\
\hline \multicolumn{4}{|l|}{ Cirrhosis } \\
\hline Yes & 20 & $0.78 \pm 0.13$ & \\
\hline No & 10 & $0.81 \pm 0.23$ & $>0.05$ \\
\hline \multicolumn{4}{|l|}{ Distant metastasis } \\
\hline Yes & 11 & $0.94 \pm 0.33$ & \\
\hline No & 19 & $0.68 \pm 0.15$ & $<0.01$ \\
\hline
\end{tabular}

western blot assays. As shown in Fig. 1A and B, the mRNA expression level of HMGB1 was significantly increased in liver cancer tissues in comparison with normal liver tissues. Also, as shown in Fig. 1C, the protein expression level of HMGB1 was also significantly increased in liver cancer tissues compared with the normal liver tissues.

Correlation of HMGB1 mRNA expression with the clinicopathologic characteristics. The relationship between HMGB1 mRNA expression and various clinical and pathologic features was analyzed. As shown in Table I, no significant correlation was found between HMGB1 expression with age, pathological type and classification, and serum AFP levels. However, the significant correlation was found between HMGB1 expression with TNM, pathological grade and distant metastasis of liver cancer.

Suppression of HMGB1 and p-AKT expression by rAd5$H M G B 1$ in SMMC-7721 cells. In order to efficiently knockdown the expression of HMGB1 in liver cancer SMMC-7721 cells, an adenovirus-mediated RNAI approach was used to construct the rAd5-HMGB1 vector. In pilot studies, the transfection efficiency of rAd5-HMGB1 (MOI=100) in SMMC-7721 cells was greater than $85.0 \%$. After rAd5-HMGB1 was transfected into SMMC-7721 cells, real-time PCR and western blot assays were performed at $48 \mathrm{~h}$ recovery to measure HMGB1 and p-AKT expression. As shown in Fig. 2A and B, an obvious inhibition of HMGB1 and p-AKT expression was observed in

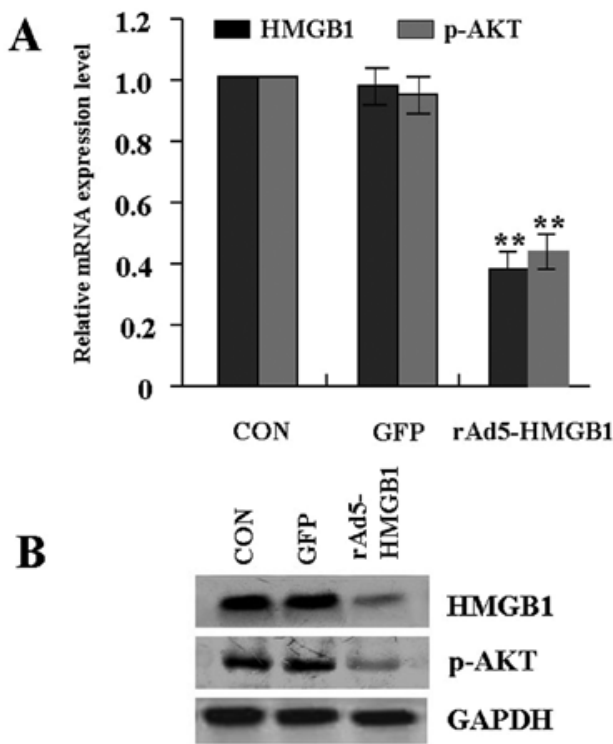

Figure 2. Suppression of HMGB1 and p-AKT expression by rAd5-HMGB1 in SMMC-7721 cells. (A and B) The expression of HMGB1 and p-AKT was examined using real-time PCR and western blot assays, and an obvious inhibition of HMGB1 and p-AKT expression was observed in rAd5-HMGB1 group compared with the GFP group and control $(\mathrm{CON})$ group $\left({ }^{* *} \mathrm{P}<0.01\right)$.

A

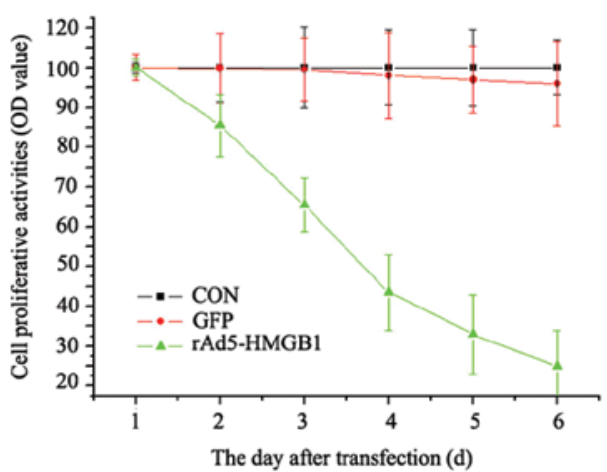

B

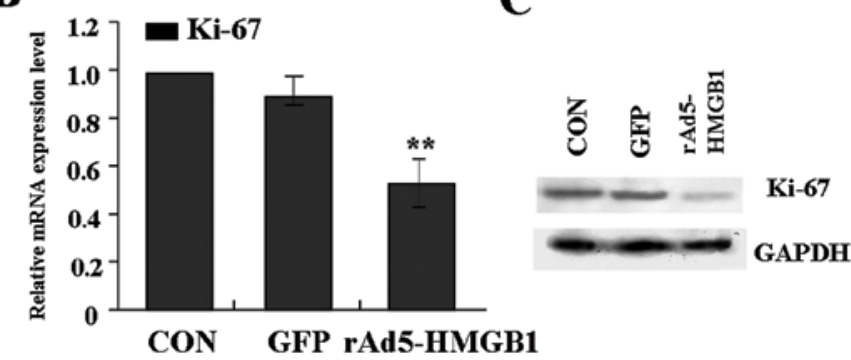

Figure 3. Suppression of liver cancer cell proliferation by rAd5-HMGB1. (A) Knockdown of HMGB1 significantly reduced the proliferative activities of SMMC-7721 cells in a time-dependent manner compared with GFP group and $\mathrm{CON}$ group. (B and $\mathrm{C}$ ) The expression of $\mathrm{Ki}-67$ was examined by realtime PCR and western blot assays. It was shown that the amount of Ki-67 expression was decreased in rAd5-HMGB1 group compared with GFP group and $\mathrm{CON}$ group $\left({ }^{* *} \mathrm{P}<0.01\right)$.

rAd5-HMGB1 group compared with the GFP group and CON group.

Suppression of liver cancer cell proliferation by rAd5-HMGB1. Deregulated cell proliferation is a hallmark of cancer (21). 
A

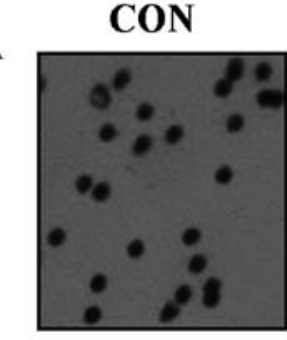

B

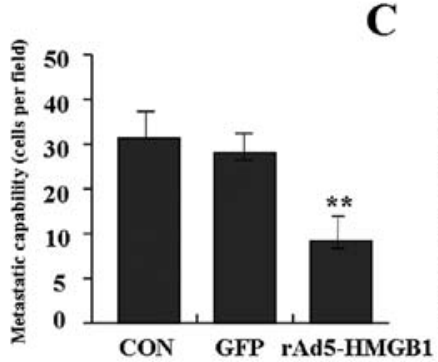

GFP

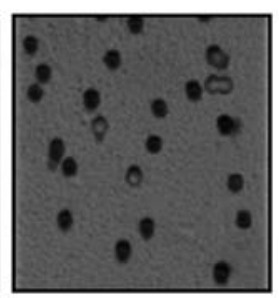

$\mathrm{C}$

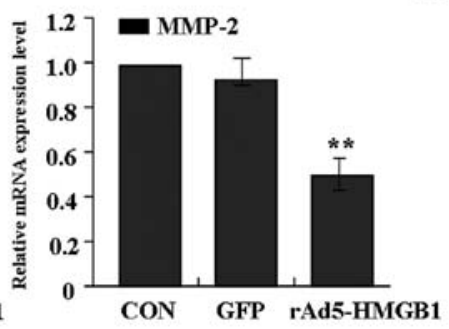

rAd5-HMGB1

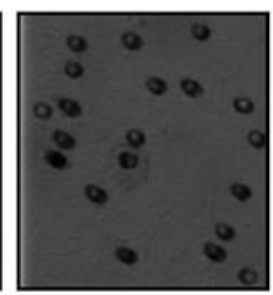

D

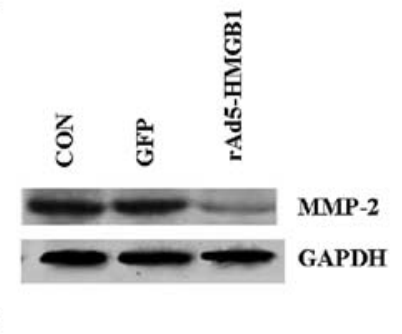

Figure 4. Suppression of liver cancer cell metastasis by rAd5-HMGB1. (A and B) The invasive metastatic capability of SMMC-7721 cells was distinctly decreased in rAd5-HMGB1 group compared with the GFP group and CON groups $\left({ }^{* *} \mathrm{P}<0.01\right)$. (C and D) The expression of MMP-2 was examined by real-time PCR and western blot assays. It was shown that the amount of MMP-2 expression was decreased in rAd5-HMGB1 group compared with GFP group and CON group, respectively $\left({ }^{* *} \mathrm{P}<0.01\right)$.

In order to test the effect of rAd5-HMGB1 on liver cancer cell proliferation, we investigated the proliferative activities of SMMC-7721 cells by MTT. As a result, it was found that knockdown of HMGB1 could significantly reduce the proliferative activities of SMMC-7721 cells in a time-dependent manner compared with GFP group and CON group (Fig. 3A). In addition, Ki-67 is at the very heart of many essential cellular processes and determines the tumor progression and the outcome of anticancer treatment. To determine whether knockdown of HMGB1 suppressed endogenous Ki-67 through translational repression, the expression of $\mathrm{Ki}-67$ was examined by Real-time PCR and western blot assays. It was shown that the amount of Ki-67 expression was decreased in rAd5-HMGB1 group compared with GFP group and $\mathrm{CON}$ group (Fig. 3B and $\mathrm{C}$ ), suggesting that knockdown of HMGB1 inhibits liver cancer cell proliferation through down-regulation of the Ki-67 expression.

Suppression of liver cancer cell metastasis by rAd5-HMGB1. To determine the effect of rAd5-HMGB1 on liver cancer cell metastasis, transwell assay was carried out. The results indicated that the invasive and metastatic potential was determined on the basis of the ability of cells to invade a matrix barrier containing laminin and type IV collagen, the major components of the basement membrane. Representative micrographs of Transwell filters can be seen in Fig. 4A. The invasive capability of SMMC7721 cells was distinctly decreased in rAd5-HMGB1 group compared with GFP group and CON group (Fig. 4B). In terms of the important role of MMP-2 in tumor metastasis, Real-time PCR and western blot assays were performed to investigate the effect of rAd5-HMGB1 on expression of MMP-2. As shown in Fig. 4C and D, the expression of MMP-2 was significantly reduced in rAd5-HMGB1 group compared with GFP group and CON group, indicating that knockdown of HMGB1 inhibits liver cancer cell metastasis through down-regulation of the MMP-2 expression.
Induction of liver cancer cell apoptosis and cycle arrest by rAd5-HMGB1. To determine whether knockdown of HMGB1 affected SMMC-7721 cell apoptosis and cycle distribution, flow cytometry with PI/FITC-Annexin V staining was performed. The results showed that the apoptotic index of SMMC-7721 cells in rAd5-HMGB1 group was markedly higher than the GFP and CON groups (Fig. 5A and C). The cycle distribution of SMMC-7721 cells was also analyzed and cell cycle kinetics showed that the $G_{0} / G_{1}$ phase fraction was increased, S-phase fraction was decreased and cell cycle was arrested in $\mathrm{G}_{0} / \mathrm{G}_{1}$ phase in $\mathrm{rAd} 5-\mathrm{HMGB} 1$ group compared with GFP and CON groups (Fig. 5B and D). Therefore, knockdown of HMGB1 can induce liver cancer cell apoptosis and block cell cycle progression.

Suppression of xenograft tumor growth by rAd5-HMGB1. Our in vitro experiments demonstrated that knockdown of HMGB1 efficiently inhibited the growth and metastasis of SMMC-7721 cells. Therefore, it is necessary to further investigate the effect of knockdown of HMGB1 on xenograft tumor growth in vivo. The mean volume of tumors in all experimental mice before treatment was $67.01 \pm 14.38 \mathrm{~mm}^{3}$. During the whole tumor growth period, the tumor growth activity was measured. Tumors treated with rAd5-HMGB1 grew relatively slowly compared with the CON and GFP groups (Fig. 6A and B). When the tumors were harvested, the average weight of tumors in group rAd5-HMGB1 was much less than that of the CON and GFP groups, respectively (Fig. 6C). This result in vivo indicated that knockdown of HMGB1 inhibited liver cancer cell growth.

\section{Discussion}

HMGB1 pathway is closely associated with tumorigenesis, expansion and invasion of multiple cancers, and plays a critical role in the development and progression of many 

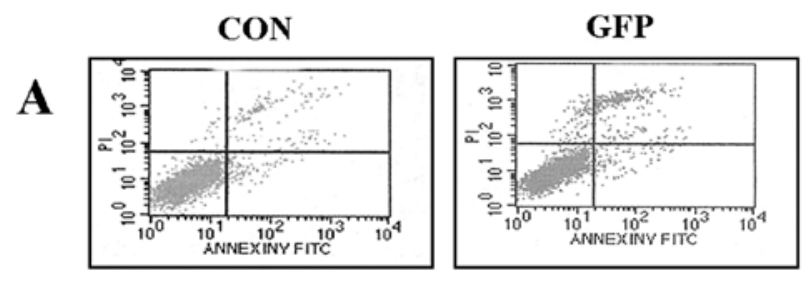

rAd5-HMGB1

B
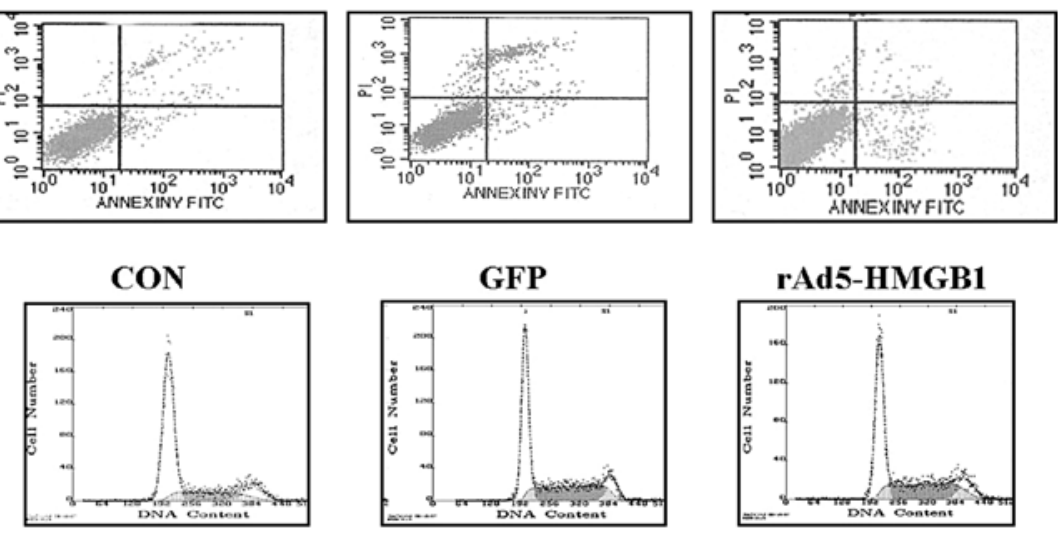

rAd5-HMGB1

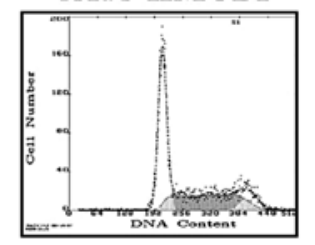

D

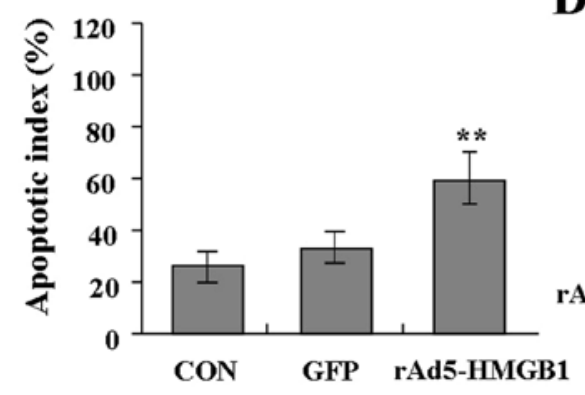

Cell cycle distribution (\%)

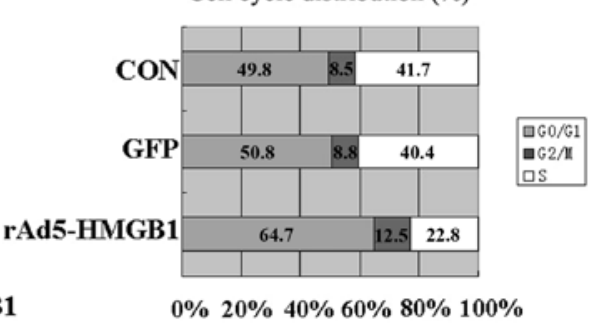

Figure 5. Induction of liver cancer cell apoptosis and cycle arrest by rAd5-HMGB1. (A and C) Cell apoptosis and cycle distribution were determined by flow cytometry with PI/FITC-Annexin V staining. The results showed that the apoptotic index of SMMC-7721 cells in rAd5-HMGB1 group was markedly higher than in the GFP and CON groups $\left({ }^{* *} \mathrm{P}<0.01\right)$. (B and D) The cycle distribution of SMMC-7721 cells was also analyzed and cell cycle kinetics showed that the $G_{0} / G_{1}$ phase fraction was increased, S-phase fraction was decreased and cell cycle was arrested in $G_{0} / G_{1}$ phase in rAd5-HMGB1 group more frequently compared with GFP and CON groups.
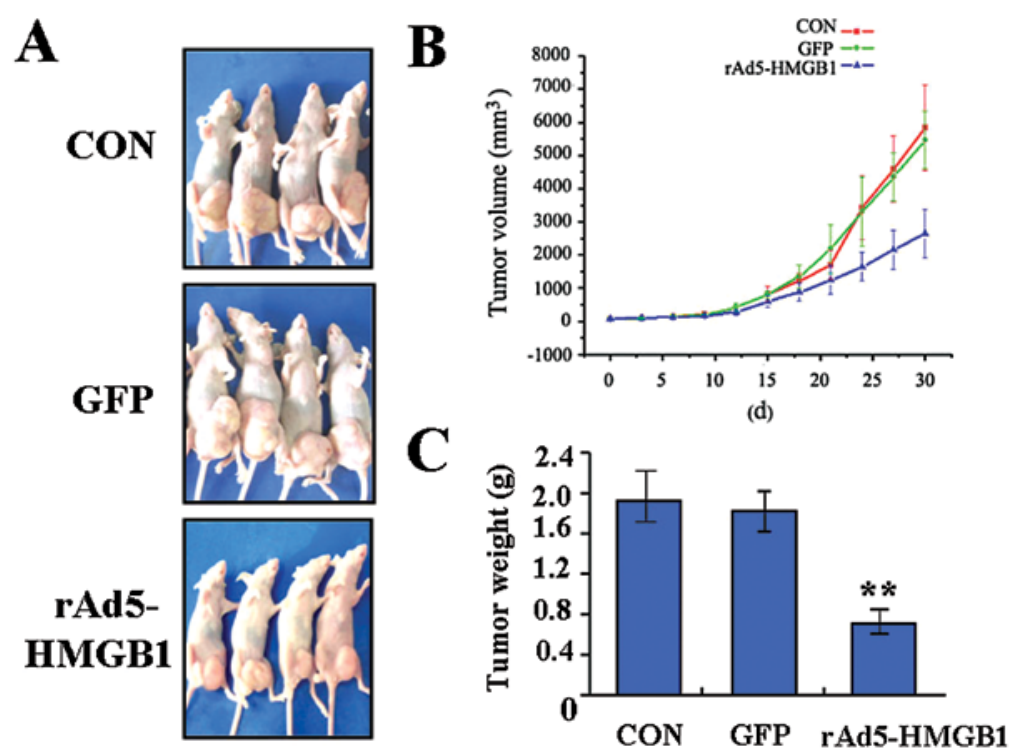

Figure 6. Suppression of xenograft tumor growth by rAd5-HMGB1. (A and B) Tumors treated with rAd5-HMGB1 grew relatively slowly compared with the CON and GFP groups. (C) When the tumors were harvested, the average weight of tumors in group rAd5-HMGB1 was much less than that of the CON and GFP group, respectively $\left({ }^{* *} \mathrm{P}<0.01\right)$.

malignant tumors. HMGB1 promotes the development and progression of renal cell carcinoma via ERK1/2 activation, which is partially mediated by RAGE (22). RAGE as a pattern recognition receptor binds HMGB1 and is involved in cancer development, progression, and metastasis (23).
Also, HMGB1 may contribute to the progression of cancer via modulation of the local immune response and promote activity of regulatory $\mathrm{T}$ cells in cancer patients $(24,25)$. Thus, HMGB1 targeting is a potential therapeutic strategy against cancer development, progression, and especially metastasis. 
Technical breakthroughs in application of HMGB1 targeting to human diseases are now urgently required (23).

Of note, some studies have found a correlation between serum HMGB1 and clinicopathological outcome of live cancer. HMGB1 may be a useful marker for evaluating the tumor stage and predicting prognosis in hepatocellular carcinoma. Targeting HMGB1 may be a potential approach for carcinoma treatment (26). In our study, it was found that HMGB1 expression was significantly increased in live cancer compared with normal live tissues, and it was also closely correlated with pathological grade and distant metastasis, representing an important therapeutic target for liver cancer.

Reduced HMGB1 expression induced by RNAI inhibits the bioactivity of hepatic carcinoma cells (20). Ethyl pyruvate is a potent inhibitor of HMGB1 release and has a therapeutic role in the treatment of cancer in conjunction with other therapeutic agents (27). To study the molecular mechanisms of HMGB1 in liver growth and metastasis, in our study, a loss of function experiment showed that knockdown of HMGB1 gene by adenovirus-mediated RNAI inhibited the proliferative activities and metastatic potential, induced cell apoptosis and cycle arrest, and slowed xenograft tumor growth in SMMC-7721 cells. Combined with the correlation of HMGB1 with pathological grade and distant metastasis of liver cancer, HMGB1 might be considered as a potential therapeutic target for liver cancer.

$\mathrm{Ki}-67$ is a nuclear protein that is expressed in proliferating cells and may be required for maintaining cell proliferation, used as a marker for cell proliferation of gastric cancer (28). MMP-2 is thought to be a key enzyme involved in the degradation of type IV collagen and high level of MMP-2 in tissues is associated with tumor growth and invasion (29). It has been reported that blockade of AKT pathway by RNAI inhibits the growth and metastasis of malignant tumors via negative regulation of Ki-67, MMP-9 and positive regulation of tissue inhibitor of metalloproteinase-2 (TIMP-2) and p53 $(30,31)$. Importantly, in our study, it was found that knockdown of HMGB1 down-regulated the expression of p-AKT, Ki-67 and MMP-2, suggesting that targeted blockade HMGB1 pathway might inhibit liver cancer growth and metastasis through AKT-mediated regulation of Ki-67 and MMP-2 expression.

In summary, the expression of HMGB1 is closely correlated with pathological grade and distant metastases of liver cancer, and targeted blockade of HMGB1 pathway inhibits liver cancer growth and metastasis in vitro and in vivo, suggesting HMGB1 may be involved in liver cancer development and progression through AKT-mediated regulation of Ki-67 and MMP-2 expression, and represent a potential therapeutic target for this aggressive malignancy.

\section{Acknowledgements}

This work was funded by Chinese National Programs for High Technology Research and Development (No. 31170938).

\section{References}

1. Jemal A, Bray F, Center MM, et al: Global cancer statistics. CA Cancer J Clin 61: 69-90, 2011.
2. Ferlay J, Shin HR, Bray F, et al: Estimates of worldwide burden of cancer in 2008: GLOBOCAN 2008. Int J Cancer 127: 2893-2917, 2010.

3. Tajima Y, Yamazaki K, Makino R, et al: Gastric and intestinal phenotypic marker expression in early differentiated-type tumors of the stomach: clinicopathologic significance and genetic background. Clin Cancer Res 12: 6469-6479, 2006.

4. Sims GP, Rowe DC, Rietdijk ST, et al: HMGB1 and RAGE in inflammation and cancer. Annu Rev Immunol 28: 367-388, 2010.

5. Okada H, Imai M, Ono F, et al: Novel complementary peptides to target molecules. Anticancer Res 31: 2511-2516, 2011.

6. Takahata R, Ono S, Tsujimoto H, et al: Postoperative serum concentrations of high mobility group box chromosomal protein-1 correlates to the duration of SIRS and pulmonary dysfunction following gastrointestinal surgery. J Surg Res 170: e135-e140, 2011.

7. Naglova $\mathrm{H}$ and Bucova M: HMGB1 and its physiological and pathological roles. Bratisl Lek Listy 113: 163-171, 2012.

8. Lee H, Shin N, Song M, et al: Analysis of nuclear high mobility group box 1 (HMGB1)-binding proteins in colon cancer cells: clustering with proteins involved in secretion and extranuclear function. J Proteome Res 9: 4661-4670, 2010

9. Tang D, Kang R, Zeh HJ III and Lotze MT: High-mobility group box 1 and cancer. Biochim Biophys Acta 1799: 131-140, 2010.

10. Kostova N, Zlateva S, Ugrinova I and Pasheva E: The expression of HMGB1 protein and its receptor RAGE in human malignant tumors. Mol Cell Biochem 337: 251-258, 2010.

11. Wu D, Ding Y, Wang S, Zhang Q and Liu L: Increased expression of high mobility group box 1 (HMGB1) is associated with progression and poor prognosis in human nasopharyngeal carcinoma. J Pathol 216: 167-175, 2008.

12. Chung HW, Lee SG, Kim H, et al: Serum high mobility group box-1 (HMGB1) is closely associated with the clinical and pathologic features of gastric cancer. J Transl Med 7: 38, 2009.

13. Liu Y, Xie C, Zhang X, et al: Elevated expression of HMGB1 in squamous-cell carcinoma of the head and neck and its clinical significance. Eur J Cancer 46: 3007-3015, 2010.

14. Choi J, Lee MK, Oh KH, et al: Interaction effect between the receptor for advanced glycation end products (RAGE) and highmobility group box-1 (HMGB-1) for the migration of a squamous cell carcinoma cell line. Tumori 97: 196-202,2011.

15. Moriwaka Y, Luo Y, Ohmori H, et al: HMGB1 attenuates antimetastatic defense of the lymph nodes in colorectal cancer. Pathobiology 77: 17-23, 2010.

16. Luo Y, Ohmori H, Fujii K, et al: HMGB1 attenuates anti-metastatic defence of the liver in colorectal cancer. Eur J Cancer 46: 791-799, 2010.

17. Yan W, Chang Y, Liang X, et al: High mobility group box 1 activates caspase- 1 and promotes hepatocellular carcinoma invasiveness and metastases. Hepatology 55: 1863-1875, 2012

18. Tafani M, Schito L, Pellegrini L, et al: Hypoxia-increased RAGE and P2X7R expression regulates tumor cell invasion through phosphorylation of Erk1/2 and Akt and nuclear translocation of NF-\{kappa\}B. Carcinogenesis 32: 1167-1175, 2011.

19. Chen J, Liu X, Zhang J and Zhao Y: Targeting HMGB1 inhibits ovarian cancer growth and metastasis by lentivirus-mediated RNA interference. J Cell Physiol 227: 3629-3638, 2012.

20. Jiang W, Wang Z, Li X, et al: Reduced high-mobility group box 1 expression induced by RNA interference inhibits the bioactivity of hepatocellular carcinoma cell line HCCLM3. Dig Dis Sci 57: 92-98, 2012.

21. Hanahan D and Weinberg RA: The hallmarks of cancer: the next generation. Cell 144: 646-674, 2011.

22. Lin L, Zhong K, Sun Z, et al: Receptor for advanced glycation end products (RAGE) partially mediates HMGB1-ERKs activation in clear cell renal cell carcinoma. J Cancer Res Clin Oncol 138: 11-22, 2012.

23. Ohmori H, Luo Y and Kuniyasu H: Non-histone nuclear factor HMGB1 as a therapeutic target in colorectal cancer. Expert Opin Ther Targets 15: 183-193, 2011.

24. Peng RQ, Wu XJ, Ding Y, et al: Co-expression of nuclear and cytoplasmic HMGB1 is inversely associated with infiltration of $\mathrm{CD} 45 \mathrm{RO}^{+} \mathrm{T}$ cells and prognosis in patients with stage IIIB colon cancer. BMC Cancer 10: 496, 2010.

25. Wild CA, Brandau S, Lotfi R, et al: HMGB1 is overexpressed in tumor cells and promotes activity of regulatory $\mathrm{T}$ cells in patients with head and neck cancer. Oral Oncol 48: 409-416 2012.

26. Cheng BQ, Jia CQ, Liu CT, et al: Serum high mobility group box chromosomal protein 1 is associated with clinicopathologic features in patients with hepatocellular carcinoma. Dig Liver Dis 40: 446-452, 2008. 
27. Liang X, Chavez AR, Schapiro NE, et al: Ethyl pyruvate administration inhibits hepatic tumor growth. J Leukoc Biol 86: 599-607, 2009.

28. Czyzewska J, Guzińska-Ustymowicz K, Lebelt A, et al: Evaluation of proliferating markers Ki-67, PCNA in gastric cancers. Rocz Akad Med Bialymst 49 (Suppl 1): 64-66, 2004.

29. Cawston TE and Wilson AJ: Understanding the role of tissue degrading enzymes and their inhibitors in development and disease. Best Pract Res Clin Rheumatol 20: 983-1002, 2006.
30. Semba S, Moriya T,Kimura W and Yamakawa M: Phosphorylated Akt/PKB controls cell growth and apoptosis in intraductal papillary-mucinous tumor and invasive ductal adenocarcinoma of the pancreas. Pancreas 26: 250-257, 2003.

31. Zhang J,Zhang QY, Fu YC, et al: Expression of p-Akt and COX-2 in gastric adenocarcinomas and adenovirus mediated Akt1 and COX-2 ShRNA suppresses SGC-7901 gastric adenocarcinoma and U251 glioma cell growth in vitro and in vivo. Technol Cancer Res Treat 8: 467-478, 2009. 